required to successfully implement these operations. Te and colleagues ${ }^{4}$ investigated the United Network of Organ Sharing database to look at outcomes in patients undergoing CHLT. ${ }^{5}$ Of 47 patients, $13 \%$ (6 patients) had CHD as a presenting diagnosis. Mortality in the whole group was $13 \%$ at 6 months, although the rate in patients with CHD was not reported. We recently reported our single institution experience with CHLT. Of 14 patients undergoing CHLT since 2008, 4 had failed single ventricle/Fontan physiology; 10 patients had biventricular failure. All 4 Fontan patients had protein-losing enteropathy with central-toportal bridging fibrosis on liver histology. The Fontan group had no mortality at 1year follow-up, whereas the biventricular failure group had 2 deaths $(20 \%)$. Outcome analysis suggested that CHLT in patients with single ventricle physiology can be performed safely, with equivocal early outcomes to CHLT in patients with biventricular failure.

\section{References}

1. Rubay JE, d'Udekem Y, Sluysmans T, Ponlot R, Jacquet L, de Laval MR. Orthotopic heart transplantation in situs inversus. Ann Thoracic Surgery. 1995;60:460-2.

2. Larsen RL, Eguchi JH, Mulla NF, Johnston JK, Fitts J, Kuhn MA, et al. Usefulness of cardiac transplantation in children with visceral heterotaxy (asplenic and polysplenic syndrome and single right-sided spleen with levocardia) and comparison of results with cardiac transplantation in children with dilated cardiomyopathy. Am J Cardiol. 2002;89:1275-9.

3. Tetsuya H, Bhama JK, Fontes PA, Toyoda Y. Combined heart and liver transplantation in a patient with situs ambiguous. Ann Thoracic Surgery. 2011;91:600-1.

4. Te HS, Anderson AS, Millis JM, Jeevanandam V, Jensen DM. Current state of combined heart-liver transplantation in the United States. J Heart Lung Transplant. 2008;27:753-9.

5. Vallabhajosyula P, Komlo C, Molina M, Roche L, Kim Y, Goldberg L, Pochettino A. 314 combined heart-liver transplantation (HLT) for failed single ventricle/Fontan physiology. J Heart Lung Transplant. 2012;31:S112.

\title{
Epicardial cryoablation resulting in precipitous left ventricular dysfunction
}

\author{
Steven M. Lampert, MD, ${ }^{a}$ Gregory F. Michaud, MD, ${ }^{b}$ Gregory S. Couper, MD, ${ }^{\mathrm{c}}$ and \\ Jochen D. Muehlschlegel, MD, MMSc, ${ }^{a}$ Boston, Mass
}

Video clip is available online.

We present a case of recurrent coronary vasospasm with subsequent hemodynamic collapse that occurred during epicardial cryoablation for refractory premature ventricular contractions (PVCs). The resulting hemodynamic changes and left ventricular dysfunction were successfully treated, leading to a favorable perioperative outcome.

\section{CLINICAL SUMMARY}

A 63-year-old man with a history of coronary artery disease and remote myocardial infarction presented with 1

\footnotetext{
From the Department of Anesthesiology, ${ }^{\mathrm{a}}$ Perioperative and Pain Medicine, Division of Cardiology, ${ }^{\mathrm{b}}$ and Division of Cardiac Surgery, ${ }^{\mathrm{c}}$ Brigham and Women's Hospital, Boston, Mass.

Disclosures: Authors have nothing to disclose with regard to commercial support.

Received for publication July 11, 2012; revisions received Dec 19, 2012; accepted for publication Jan 11, 2013; available ahead of print Jan 31, 2013.

Address for reprints: Jochen D. Muehlschlegel, MD, MMSc, Department of Anesthesiology, Perioperative and Pain Medicine, Brigham and Women's Hospital, 75

Francis St, Boston, MA 02115 (E-mail: jmuehlschlegel@ partners.org).

J Thorac Cardiovasc Surg 2013;145:e41-2

$0022-5223 / \$ 36.00$

Copyright (C) 2013 by The American Association for Thoracic Surgery

http://dx.doi.org/10.1016/j.jtcvs.2013.01.023
}

year of palpitations, dyspnea on exertion, and multiple syncopes. Holter monitoring demonstrated frequent PVCs. Preoperative transesophageal echocardiography (TEE) showed normal valvular function, preserved ejection fraction, and left ventricular hypertrophy without wall motion abnormalities. Although 2 separate endocardial ablation procedures temporarily terminated the premature beats, PVCs persisted and medical management ultimately failed, which prompted off-pump epicardial cryoablation of the left ventricular myocardium. Both radial arterial line and internal jugular triple lumen catheters were placed followed by a standard intravenous induction and maintenance anesthesia using isoflurane, and a TEE probe was placed for intraoperative monitoring. An isoproterenol infusion was used to augment the PVCs until a regular frequency was established. Electrophysiology studies showed that the origin of the PVCs was located on the anterobasal left ventricular epicardial surface near the bifurcation of the left main coronary artery (Figure 1). The area of interest was close to the origin of the anterior cardiac vein from the great cardiac vein. A full sternotomy was performed, the pericardium was opened, and dissection to the area of interest was uneventful. Mobilization and manipulation of the coronary arteries were performed to target the areas of interest. The line of ablations of the cryoprobe (Cardioblate CryoFlex; Medtronic ATS Medical Inc, Minneapolis, Minn) was alongside 


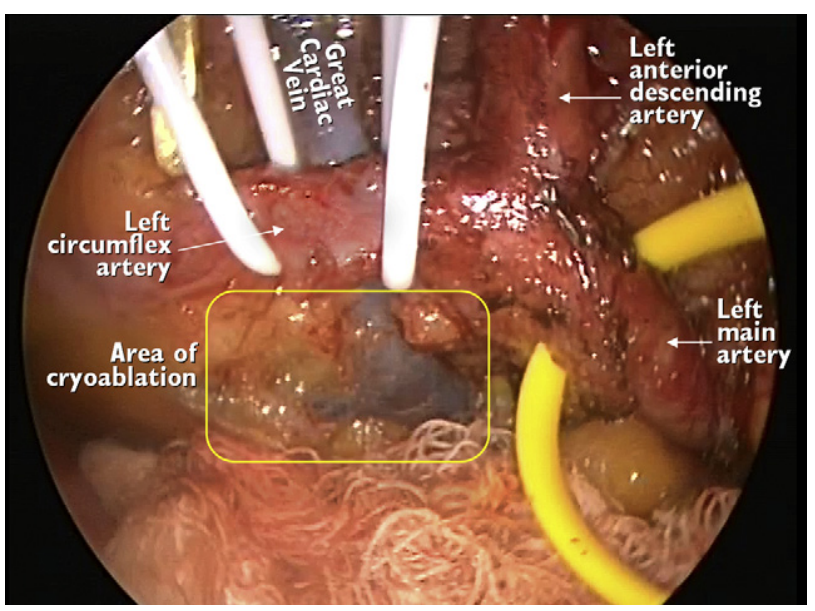

FIGURE 1. Site of epicardial cryoablation.

and underneath the anterior cardiac vein, underneath the left main, the bifurcation, adjacent to the left anterior descending artery, and underneath the proximal circumflex artery. Cryoablations from the basal myocardium almost to the base of the left atrial appendage apically were performed with multiple 5-minute cooling periods (to $-150^{\circ} \mathrm{C}$ ). During these cooling periods, we noted the immediate development of greater than 5-mm ST-segment elevations in the anterior and lateral leads, bradycardia, hypotension, and anterior and lateral wall hypokinesis on TEE imaging (left ventricular ejection fraction from $60 \%$ to $10 \%$ ), which lasted 3 to 4 minutes (Video 1). Recovery to baseline was achieved with vasopressor support (intravenous boluses of epinephrine and phenylephrine), topical normal saline and papaverine sprayed directly onto the coronary arteries, discontinuation of the cooling cycle, and limited surgical manipulation. During the cooling periods, the PVCs would stop but subsequently returned during the rewarming phase. After multiple unsuccessful cryoablation cycles, the procedure was terminated. The patient recovered without perioperative complications, although an immediate postoperative angiogram was not done. Several months after the procedure, exertional chest pain developed in this patient with known coronary artery disease and previous myocardial infarction. An angiogram revealed narrowing in the left anterior descending at the site of cryoablation that was subsequently treated with a drug-eluting stent. A follow-up angiogram showed open coronary arteries.

\section{DISCUSSION}

Although off-pump surgical epicardial cryoablation is currently a relatively uncommon approach to arrhythmia management, this procedure provides an opportunity to control refractory arrhythmias that have failed more conservative management, albeit not without risks. One theoretic advantage of cryoenergy is the reduced risk of damage to adjacent structures, such as the coronary arteries, when compared with other ablation techniques. ${ }^{1}$

In the described case, activation of the cryoprobe was preceded temporally to each hemodynamic event. Coronary vasospasm as a direct result of cryoablation and surgical manipulation adjacent to the coronary arteries led to near hemodynamic collapse. During each episode, the patient responded favorably and rapidly to vasopressor support, topical papaverine, and discontinuation of both the cooling and surgical manipulation. Acute hemodynamic collapse occurred predictably with every subsequent cryoablation. Anticipating this left ventricular dysfunction during subsequent cryoablation cycles allowed more rapid response and shortened episodes of hypotension, bradycardia, ST changes, and left ventricular dysfunction. Unfortunately, this patient's PVCs continued both intraoperatively and postoperatively despite repeated attempts at cryoablation. Nevertheless, knowledge of the relevant anatomy, assisted by real-time TEE imaging, and vigilance and preemptive treatment during subsequent cryoablations allowed a favorable hemodynamic recovery with resolution of echocardiographic and ST-segment abnormalities.

\section{CONCLUSIONS}

On the basis of our experience in this case and consistent with previously published guidelines, ${ }^{2}$ continuous TEE monitoring should be strongly considered. Furthermore, we advocate for in-line, readily available, vasopressor support before ablation or surgical manipulation near the coronaries. Last, we found that open communication among the anesthesiologist, cardiac surgeon, electrophysiologist, and other members of the operating team allowed for optimal patient outcome.

\section{References}

1. Di Biase L, Al-Ahamad A, Santangeli P, et al. Safety and outcomes of cryoablation for ventricular tachyarrhythmias: results from a multicenter experience. Heart Rhythm. 2011;8:968-74.

2. Thys DM, Brooker RF, Cahalan MK, et al. Practice guidelines for perioperative transesophageal echocardiography. An updated report by the American Society of Anesthesiologists and the Society of Cardiovascular Anesthesiologists Task Force on Transesophageal Echocardiography. Anesthesiology. 2010;112:1084-96. 